\title{
Modelado e implementación computacional de la propagación de las enfermedades de tranmisión sexual por autómatas celulares (Cell-DEVS)
}

Modelling and computational implementation of the spread of sexually transmitted diseases by cellular automata (Cell-DEVS)

Recibido: marzo 05 de 2020 | Revisado: octubre 02 de 2020 | Aceptado: enero 20 de 2021

Neisser Pino Romero ${ }^{\mathrm{I}}$

GABRIEL WAineR $^{2}$

1 Departamento de Ciencias Exactas, Facultad de Ciencias Exactas, Universidad Peruana Cayetano Heredia, Lima, Perú

2 Departament of Systems and Computer Engineering, Carleton University, Ottawa, Canada

Autor de correspondencia:

E-mail: neisser.pino@upch.pe

\begin{abstract}
Resumen
En este trabajo se realizó un modelo computacional a través de los Autómatas Celulares que puede describir la dinámica de cómo se propagan las enfermedades de transmisión de sexual considerando una subclasificación para la población de infectados (curable y no curable). Así mismo, se ha considerado en este trabajo la perspectiva de la epidemiología matemática del modelo matemático SIS de W.O. Kermack y A.G. McKendrick que representa la dinámica de la epidemia, siendo posible así analizar el comportamiento de la propagación de la epidemia en el tiempo.
\end{abstract}

Palabras clave: Epidemiología Matemática, ecuaciones diferenciales, autómatas celulares, formalismo DEVS, simulaciones computacionales

\begin{abstract}
In this work, we constructed a computational model by Cellular Automatas (Cell-DEVS) that can describe the dynamics of how sexually transmitted diseases (STD) spread considering a subclassification for the Infected population (curable STD and non-curable STD). Furthermore, we consider the mathematical epidemiology mathematical the model SIS of W.O. Kermack and A.G. McKendrick that represents the dynamics of the epidemic (STD), therefore we can analyze the behavior of the spread of the epidemic over time.
\end{abstract}

Key words: Mathematical Epidemiology, differential equations, cellular automata, DEVS formalism, computational simulations

\footnotetext{
(C) Los autores. Este artículo es publicado por la Revista Campus de la Facultad de Ingeniería y Arquitectura de la Universidad de San Martín de Porres. Este artículo se distribuye en los términos de la Licencia Creative Commons Atribución No-comercial - Compartir-Igual 4.0 Internacional (https://creativecommons.org/licenses/ CC-BY), que permite el uso no comercial, distribución y reproducción en cualquier medio siempre que la obra original sea debidamente citada. Para uso comercial contactar a: revistacampus@usmp.pe.
} 


\section{Introducción}

En 1927, W. O. Kermack y A. G. McKendrick propusieron un modelo matemático que intentaba describir el comportamiento de las enfermedades infecciosas (Anderson, 1982), donde se comenzó a recurrir a la formulación de modelos matemáticos para intentar dar solución a diferentes fenómenos en el área de la salud en el cual únicamente se considera el tiempo (ecuaciones diferenciales ordinarias) como lo indica Trottier y Philippe (2000) y con la validación de los datos que proporcionaba las instituciones de salud (Ministerio de Salud, Organización Mundial de la Salud, etc.). De forma similar, se realizó un modelamiento mediante las ecuaciones diferenciales parciales, donde se considera el tiempo-espacio que podría describir la difusión de la epidemia a través del contacto espacial mediante el tiempo de considerado (Elkadry, 2013).

En ese contexto, la computación comenzó a ser una herramienta fundamental para poder procesar grandes cantidades de datos. Desde esta perspectiva, los métodos numéricos ofrecen una aproximación de soluciones mediante simulaciones computacionales de un sistema dinámico (Tiempo Discreto o Eventos Discretos) como lo realizan Kofman (2001), Wainer y Castro (2010). En los últimos años, los autómatas celulares han permitido representar de una manera más sencilla muchos sistemas dinámicos de una gran complejidad por medio de reglas de transición (Eventos Discretos). (Wainer y Giambiasi, 2001).

Según el Centro Nacional de Epidemiología, Prevención y Control de
Enfermedades y el "National Institute of Health" (NIH), las enfermedades de transmisión sexual (ETS) se podrían clasificar de diversas formas. Entre las enfermedades más conocidas que no tienen cura son: VIH, el herpes genital, el virus del papiloma humano, y la hepatitis. Y de las que tienen cura son: gonorrea, clamidia, sífilis, tricomona entre otras. El único medio de contagio de la ETS es por la vía sexual debido que es el principal medio de contagio como indica la Organización Mundial de la Salud. Además, un individuo puede poseer más de una ET, en este caso, se necesita un estudio más detallado para mantener un nivel bajo la prevalencia y el contagio de las ETS, objetivo principal de las Instituciones de Salud Pública. Estas consideraciones las referencian Anderson (1982) y Cueto (2001) y mucha de la teoría matemática aplicada a la epidemiología se ve en el libro de Brauer y Castillo (2001).

\section{Modelo matemático SEIRS}

El modelo matemático SEIRS (Susceptible, Expuesto, Infectado, Recuperado) es uno de los modelos epidemiológicos (Anderson, 1982). Para nuestro caso, consideraremos el modelo SEIRS con tres subpoblaciones de infectadas (ETS curable, ETS no curable, y a la vez, los que poseen ambas). Esta problemática se puede formular el modelo mediante las ecuaciones diferenciales ordinarias como se expresa en Pino (2017), con toda la base de los datos y las directrices que brinda las instituciones nacionales como la Dirección Técnica de Demografía e Indicadores Sociales del Ministerio de Salud y la Oficina de Estadística e Informática (INEI) y a la vez del Instituto Nacional de Salud (INS). 


\section{Modelo matemático SIS}

Para la dinámica de la ETS, consideramos las poblaciones de susceptibles y de infectados. La población de infectados se dividió en tres poblaciones donde se encuentran los individuos diferenciados por la dimensión de su enfermedad (ETS Curable, ETS No Curable, y la que posee ambas ETS (curable y no curable)) como se referencia en los informes técnicos de la OMS (2016) y del Centro Nacional de Epidemiología, Prevención y Control de Enfermedades (2020). No tomaremos el periodo de incubación de la enfermedad (población de expuestos). Tampoco se toma en cuenta la población de recuperados debido a que consideramos que los infectados que se recuperen son inmediatamente la población de los susceptibles (Pino et al. 2018) y (Brauer y Castillo, 2001).

Este modelo no considera natalidad ni mortalidad; es decir, ingreso o salida de población de ningún tipo. Y esta hipótesis matemáticamente se representa con la ecuación:

$$
N(t)=S(t)+I_{c}(t)+I_{n c}(t)+I_{x}(t)
$$

constante (Trottier y Philippe, 2000). Las variables (poblaciones epidemiológicas) para nuestro modelo son los siguientes:

- $S(t) \quad$ : Población de los Susceptibles en el tiempo ( $t$ )

- $I_{c}(t)$ : Población de los Infectados (ETS Curable) en el tiempo ( $t$ )

- $I_{n c}(t)$ : Población de los Infectados (ETS No Curable) en el tiempo (t)

- $I_{x}(t)$ : Población de los Infectados (posee ambas ETS) en el tiempo $(t)$

Los parámetros (tasas epidemiológicas) para nuestro modelo son los siguientes:

- $\quad \beta_{1}$ : Tasa de contagio entre un Susceptible $(S)$ y un Infectado $\left(I_{c}(t)\right)$

- $\beta_{2}$ : Tasa de contagio entre un Susceptible $(S)$ y un Infectado $\left(I_{n c}(t)\right)$

- $\varphi_{1}$ : Tasa de contagio entre un Infectado $\left(I_{c}(t)\right)$ y un Infectado $\left(I_{n c}(t)\right)$

- $\varphi_{2}$ : Tasa de contagio entre un Infectado $\left(I_{n c}(t)\right)$ y un Infectado $\left(I_{C}(t)\right)$

- $\omega_{1}$ : Tasa de recuperación de un Infectado $\left(I_{\mathcal{C}}(t)\right)$

- $\omega_{2}$ : Tasa de recuperación de un Infectado $\left(I_{x}(t)\right)$

Teniendolas presentes consideraciones, el modelo matemático modela la interacción de las poblaciones mediante

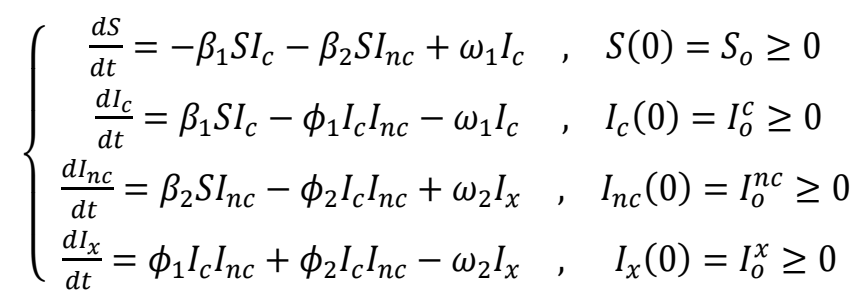

Este modelo matemático representa la propagación de la ETS. Este sistema está bien definido con lo cual se puede garantizar la existencia y unicidad de las soluciones, además la positividad de las soluciones que garantizan la no sus tasas de contacto en Anderson (1982), Brauer y Castillo (2001) y Pino (2017).

negatividad de las poblaciones por el contrario se centra en la simulación computacional que proviene de nuestro sistema de ecuaciones (Pino et al., 2018 y Reynaga, 2015). 


\section{Simulación computacional}

La simulación computacional se basa en el método de Runge-Kutta de orden cuatro mediante la discretización del tiempo. La simulación también se pudo realizar mediante eventos discretos (DEVS) (Kofman, 2001) y consideramos las poblaciones iniciales y las tasas epidemiológicas correspondientes. Se determinó un tiempo de simulación de 60

Tabla 1

Poblaciones epidemiológicas

\begin{tabular}{lc}
\hline \multicolumn{1}{c}{ Población epidemiológica } & $\begin{array}{c}\text { Población } \\
\text { inicial }\end{array}$ \\
\hline Susceptibles & 4940 \\
Infectados con ETS Curable & 45 \\
Infectados con ETS No Curable & 15 \\
Infectados con ambas ETS & 0 \\
\hline
\end{tabular}

Las tasas epidemiológicas, en valores normalizados que, según los datos históricos del Centro Nacional de Epidemiología, Prevención y Control de Enfermedades permiten evaluar la evolución temporal de la enfermedad son las siguientes: (meses) para analizar el comportamiento de cada población. Aquí, las poblaciones iniciales consideradas para nuestra simulación son las siguientes que se han adaptado de los reportes brindados por el Centro Nacional de Epidemiología, Prevención y Control de Enfermedades y el "National Institute of Health" que nos permiten ver cómo se puede generar la simulación computacional para nuestro modelo.

Tabla 2

\begin{tabular}{cc} 
Tasas epidemiológicas & \\
\hline $\begin{array}{c}\text { Tasa } \\
\text { Epidemiológica }\end{array}$ & $\begin{array}{c}\text { Valor } \\
\text { Normalizado }\end{array}$ \\
\hline$\beta_{1}$ & 0.15 \\
$\beta_{2}$ & 0.08 \\
$\phi_{1}$ & 0.25 \\
$\phi_{2}$ & 0.15 \\
$\omega_{1}$ & 0.30 \\
$\omega_{2}$ & 0.20 \\
\hline
\end{tabular}

De acuerdo a las Tabla 1 y 2 se obtiene la siguiente simulación sobre la propagación de la enfermedad de trasmisión sexual (ETS).

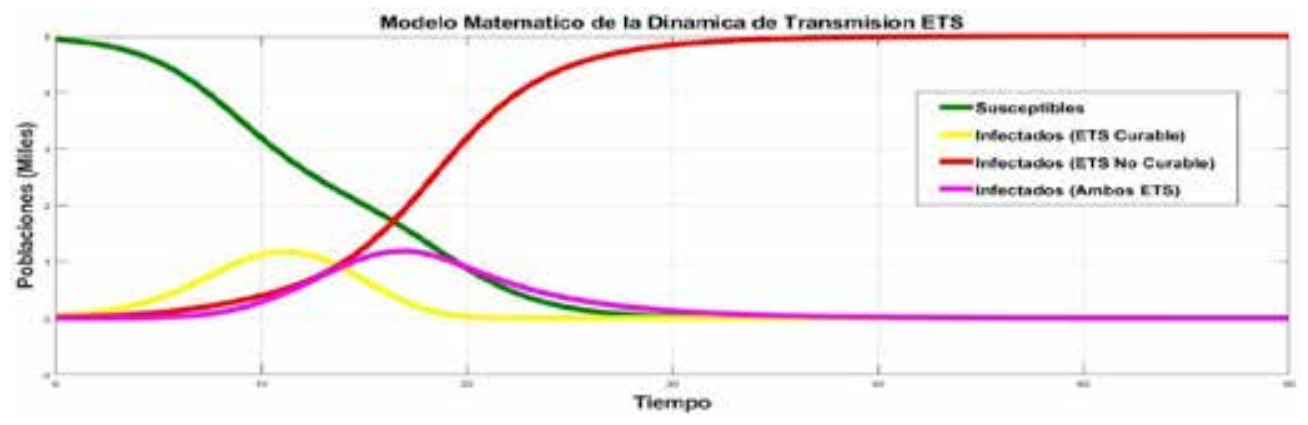

Figura 1. Simulación del Modelo Matemático

La dinámica de la evolución de las poblaciones describe que con el pasar del tiempo, los Susceptibles van descendiendo, mientras que los Infectados van aumentando y en el largo plazo la subpoblación de Infectados que poseen una ETS No curable va obteniendo una mayor presencia en la población total hasta que ocupe toda la población como lo indica el Boletín Estadístico sobre Infecciones sobre Infecciones de Transmisión Sexual Perú: 2002-2011 (Ministerio de Salud, 2012). Este detalle es fácil ver debido que estos infectados 
siempre permanecen infectados en el tiempo. Las otras dos subpoblaciones de infectados e tienen brotes significativos debido que los infectados con ETS curable pasan a la población susceptible, pero continúan expuestos a contraer de nuevo la enfermedad. Por otro lado, los infectados con ambas ETS se pueden recuperar de la ETS curable pero siempre permanecerán con la ETS (no curable) en el tiempo. Estas observaciones se dan en el Centro Nacional de Epidemiología, Prevención y Control de Enfermedades debido a que no hay mejor método de protección ante esta enfermedad que la prevención y la comunicación debida de la enfermedad a la sociedad. (Cueto, 2001 y Pino, 2017).

\section{Formalismo DEVS}

El autómata celular es un modelo matemático para un sistema dinámico, conformado por un conjunto de celdas o células que adquieran distintos estados o valores. Estos estados son alterados de un instante a otro en unidades de tiempo discreto; es decir, que se puede cuantificar con valores enteros a intervalos regulares. (Wainer y Mosterman, 2016 y Wainer, 2018). Una vez que se define el comportamiento de una sola célula, se necesita formar un espacio celular (malla). Se incluye la definición de los modelos acoplados a dos dimensiones de Cell-DEVS con vecinos adyacentes. La técnica de modelamiento mediante los Autómatas Celulares permite mantener la capacidad para describir sistemas complejos utilizando reglas muy sencillas, al mismo tiempo que nos permite cerrar la brecha entre un tiempo discreto y una descripción de evento discreto (Wainer, 2018).

\section{Formalización del modelo SIS}

El modelo SIS (SusceptiblesInfectados-Susceptibles), y luego, donde las reglas correspondientes para poder generar la simulación desde la perspectiva de los Autómatas Celulares (Cell-DEVS). (Wainer y Mosterman, 2016).

Epidemia $=<X, Y, D,\left\{M_{i}\right\},\left\{I_{i}\right\},\left\{Z_{i}\right\}$, select $>$

- $X=\varnothing$

- $Y=\varnothing$

- $D=\{$ cell $\}$

- $M_{\text {cell }}=$ Cell_SIS

- $I_{\text {cell }}=\varnothing$

- $Z_{i}=\varnothing$

- $\quad$ select $=($ Cell_SIS $)$

Cell_SIS $=<X_{\text {list }}, Y_{\text {list }}, I, X, Y, n,\left\{t_{1}, t_{2}, \cdots, t_{n}\right\}, \eta, N, C, B, Z$, select $>$

- $X_{\text {list }}=\emptyset$

- $Y_{\text {list }}=\varnothing$

- $I=<p^{x}, p^{y}>$ donde: $p^{x}=p^{y}=\varnothing$

- $X=\{0,1,2,3\}$

- $n=2$ 
- $\eta=2$

- $t_{1}=t_{2}=10$

- $\quad N=\{(-1,-1),(-1,0),(-1,1),(0,-1),(0,0),(0,1),(1,-1),(1,0),(1,1)\}$

- $B=\emptyset$

- $Z=\left\{P_{i, j} Y_{1} \rightarrow P_{i, j-1} X_{1}, P_{i, j} Y_{2} \rightarrow P_{i+1, j} X_{2}, P_{i, j} Y_{3} \rightarrow P_{i, j+1} X_{3}, P_{i, j} Y_{4} \rightarrow\right.$ $P_{i-1, j} X_{4}, P_{i, j} Y_{5} \rightarrow P_{i, j} X_{5}, P_{i, j+1} Y_{1} \rightarrow P_{i, j} X_{1}, P_{i-1, j} Y_{2} \rightarrow P_{i, j} X_{2}, P_{i, j-1} Y_{3} \rightarrow$ $\left.P_{i, j-1} X_{3}, P_{i+1, j} Y_{4} \rightarrow P_{i, j} X_{4}, P_{i, j} Y_{5} \rightarrow P_{i, j} X_{5}\right\}$

$\boldsymbol{C}_{i, j}=<, I, X, Y, N, \boldsymbol{\delta}_{\text {int }}, \boldsymbol{\delta}_{\text {ext }}, \boldsymbol{d}, \boldsymbol{\tau}, \lambda>$

- $X_{\text {list }}=\emptyset$

- $Y_{\text {list }}=\varnothing$

- $p^{x}=\left\{X_{k} \in\{0,1,2,3\}\right.$ donde $\left.k=\overline{1,9}\right\}$

- $p^{y}=\left\{X_{k} \in\{0,1,2,3\}\right.$ donde $\left.k=\overline{1,9}\right\}$

- $X=\{0,1,2\}$

- $Y=\{0.1 .2\}$

- $\quad N=\{(-1,-1),(-1,0),(-1,1),(0,-1),(0,0),(0,1),(1,-1),(1,0),(1,1)\}$

- $d=$ Tiempo(semanas)

- $\quad S X$ : Variables Descriptivas

$$
S X= \begin{cases}0 & : \text { Persona Susceptible } \\ 1 & : \text { Persona Infectada con ETS Curable } \\ 2 & : \text { Persona Infectada con ETS No Curable } \\ 3 & : \text { Persona Infectada con ambas ETS }\end{cases}
$$

El modelo computacional indica el color de la célula que representa la población epidemiológica y pueda visualizarse su evolución en el tiempo.

$$
S X=\left\{\begin{array}{lll}
0 & : & \text { Color Verde } \\
1 & : & \text { Color Amarillo } \\
2 & : & \text { Color Rojo } \\
3 & : & \text { Color Rosado }
\end{array}\right.
$$

\section{Reglas del modelo computacional}

Las reglas definen la dinámica de transición de los diferentes estados de las células. La regla para el modelo computacional es similar a las hipótesis que del modelo matemático ecuaciones diferencias ordinarias. La esencia del modelo computacional son las reglas que definen la transición de las celdas, como en el modelo matemático son las consideraciones. Para la simulación computacional del modelo, con un vecindario Neumann de radio 1 , donde la celda central se relaciona con ocho celdas a su alrededor, la frontera "wrapped"; es decir, las celdas de la frontera también interactúan con ocho celdas para su proceso de transición (tiempo y espacio) de tal forma que genere su evolución respectiva en el tiempo de simulación. (Bonaventura et al., 2013) y (Wainer y Mosterman, 2016).

La expresión $\mathrm{Pb}$ ( ) es el elemento de una regla que permite cuantificar la 
influencia que generan las celdas vecinas para ocasionar un cambio de transición. Este detalle se asemeja a los valores de las tasas epidemiológicas (Ministerio de Salud, 2015; NIH, 2019 y OMS, 2016). La diferencia entre dos datos es la cantidad que contactos que tiene cada individuo.
En el modelo matemático comprende la probabilidad de un individuo al entrar con todas las poblaciones; mientras que el modelo computacional solamente comprende el vecindario considerado. (Ministerio de Salud, 2006 y Wainer, 2018).

Tabla 3

Reglas de transición del modelo computacional

\begin{tabular}{ccc}
\hline $\begin{array}{c}\text { Estado inicial de } \\
\text { la célula }\end{array}$ & $\begin{array}{c}\text { Regla para que se realice el cambio de } \\
\text { estado }\end{array}$ & $\begin{array}{c}\text { Estado final de } \\
\text { la célula }\end{array}$ \\
\hline 0 & $\#(2) \geq 1 \wedge \#(1)>\#(2) \wedge P b\left(\beta_{1}\right)$ & 1 \\
0 & $\#(2) \geq 1 \wedge \#(2)>\#(1) \wedge P b\left(\beta_{2}\right)$ & 2 \\
1 & $\#(2)+\#(3)=0 \wedge P b\left(\omega_{1}\right)$ & 0 \\
1 & $\#(3) \geq 1 \wedge P b\left(\phi_{1}\right)$ & 3 \\
2 & $\#(3) \geq 1 \wedge P b\left(\phi_{2}\right)$ & 3 \\
3 & $\#(2)+\#(3)=0 \wedge P b\left(\omega_{2}\right)$ & 2 \\
\hline
\end{tabular}

\section{Simulación computacional}

De una forma similar, se considera las poblaciones iniciales para la simulación del modelo computacional (autómatas celulares) como se realizó con el modelo

Tabla 4

Poblaciones epidemiológicas

\begin{tabular}{lc}
\hline \multicolumn{1}{c}{ Población epidemiológica } & Población inicial \\
\hline Susceptibles $(S)$ & 2250 \\
Infectados con ETS Curable $\left(I_{c}\right)$ & 158 \\
Infectados con ETS No Curable $\left(I_{n c}\right)$ & 66 \\
Infectados con ambas ETS $\left(I_{x}\right)$ & 26 \\
\hline
\end{tabular}

Se emplea una malla cuadrangular de (2500 células) ubicada en las distintas poblaciones epidemiológicas. La simulación computacional mediante los autómatas celulares nos muestra cómo se propaga la epidemia considerando el tiempo y el espacio. (Pino et al., 2018 y Wainer, 2018). La posición inicial dentro de la malla es de forma estructurada para poder realizar la simulación y distribuida por los datos de la Guía reportados por el Ministerio de Salud (2012 y 2015). matemático (métodos numéricos). La simulación computacional se implementó en $\mathrm{CD}++$ (Bonaventura et al., 2013) y las poblaciones iniciales son mostradas en la Tabla 4.
La simulación es dentro del periodo considerado para poder analizar la evolución de la enfermedad en tiempo y espacio. (Wainer y Mosterman, 2016). La selección de posición con respecto a las células se debe a una distribución normal de acuerdo al reporte generado por el Ministerio de Salud (2015) que nos permite tener un análisis en cierto modo aleatorio y cómo ubicar focos de infección para poder realizar una intervención epidemiológica y reducir contagios. 


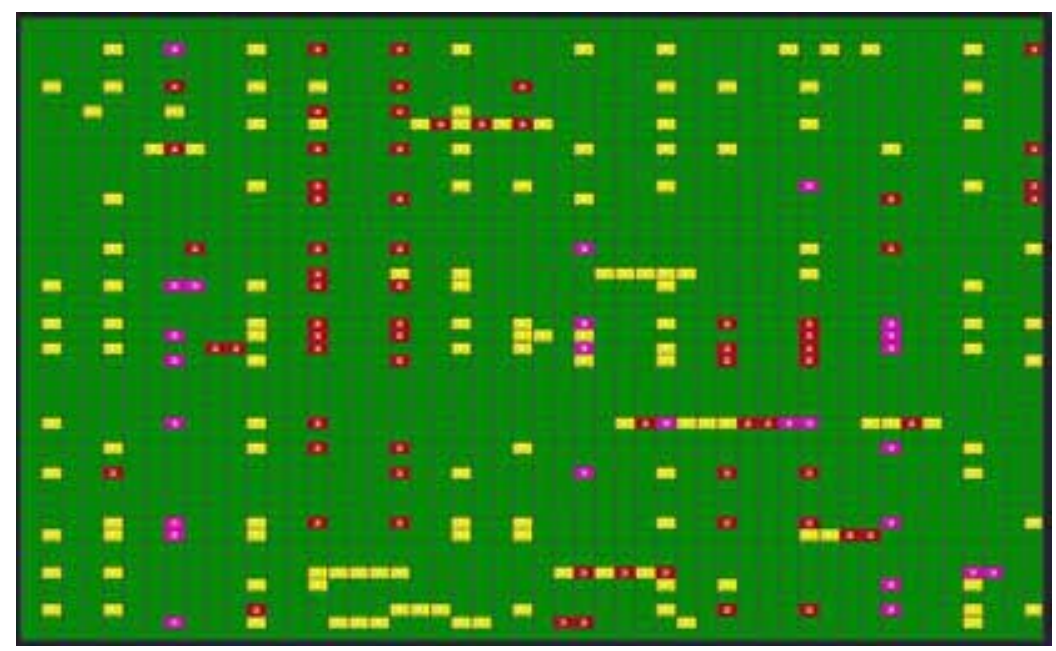

Figura 2. Estado inicial del Modelo Computacional

De este estado inicial, se comenzó a propagar la enfermedad bajo las reglas de transición definidas. Dependiendo de la posición de cada celda y la interacción con su vecindario evoluciona la epidemia de la ETS. (Wainer, 1998 y Pino et al., 2018).

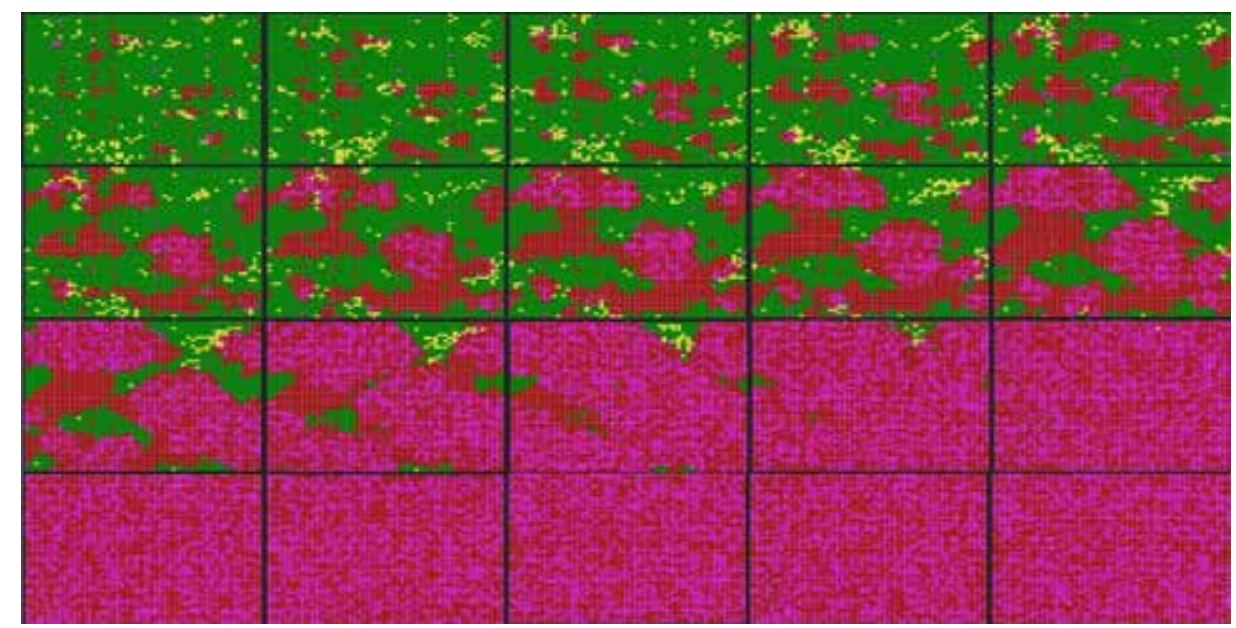

Figura 3. Evolución del Modelo Computacional en Tiempo y Espacio

La simulación computacional describe cómo la epidemia de las ETS se va propagando en el tiempo y espacio, además donde las poblaciones infectadas van creciendo, especialmente, la población con ETS No curable y la población que posee ambas ETS . Este comportamiento es clásico en los modelos de epidemias en cuanto a su propagación en el tiempo de una manera más real que en la primera simulación mediante el modelo matemático debido a la interacción que tiene con el vecindario considerado en el modelo computacional (Anderson, 1982 y Cueto, 2001). De la simulación generada por los autómatas celulares, se puede realizar una gráfica (ploteo) de las poblaciones epidemiológicas en el tiempo para analizar su comportamiento. Esto permite contrastar con la simulación del modelo matemático (ecuaciones diferenciales). (Pino et al., 2018). 


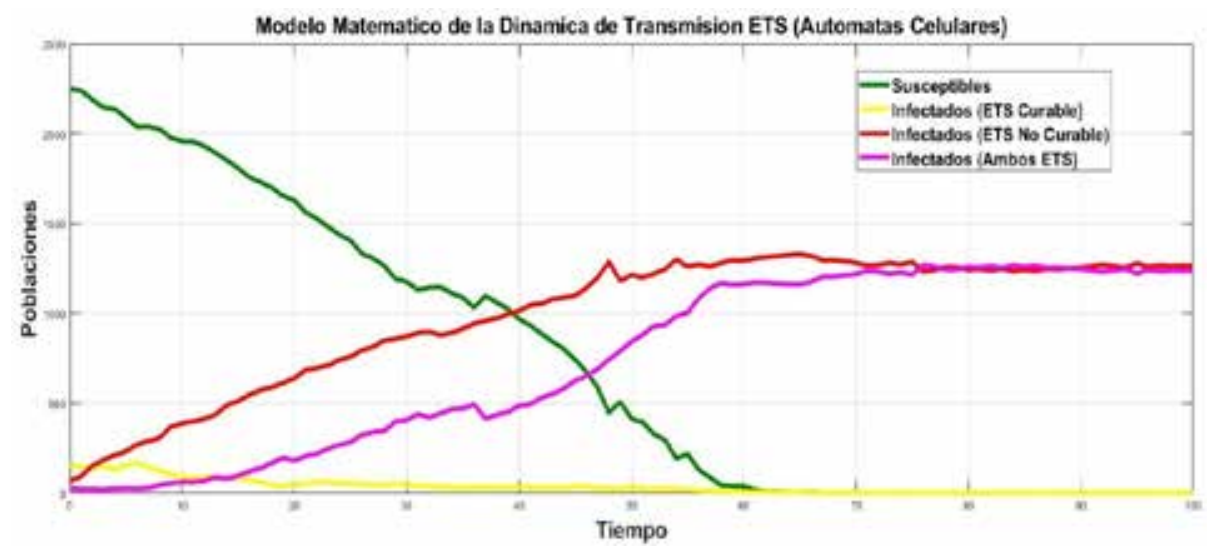

Figura 4. Evolución del Modelo Computacional en el tiempo

Después de obtener las curvas de las poblaciones, se observa que la población susceptible disminuye mientras que las poblaciones de infectados incrementan. El detalle es que en los infectados con ETS curable, su crecimiento es leve para luego descender. Esto ocurre debido a la interacción con los otros infectados tienen mayor incidencia y permanencia (OMS, 2016). La mayor diferencia que se tiene con la simulación mediante las ecuaciones diferenciales es la población de los Infectados con ETS No curable que crece de tal forma hasta posicionarse como la población total, lo cual en la vida real no se da. (Ministerio de Salud, 2012 y 2015). Este hecho no se representa en la simulación mediante autómatas celulares debido que hay una convivencia entre los infectados con ETS No curable y de los que poseen ambas ETS en el tiempo (INS, 2015). Este detalle lo explica el hecho de la densidad de contacto que tiene un individuo con respecto a la población; es decir, en las ecuaciones diferenciales un individuo se relaciona con todos los individuos con lo cual genera una mayor probabilidad de contagiarse (tasa de contagio) por mientras que en los autómatas celulares el vecindario definido determina la interacción que tienen los individuos entre sí. (Wainer, 2002 y 2018).

\section{Perturbación del modelo computacional}

Siempre se realizan perturbaciones a los modelos matemáticos para que el nuevo modelo pueda reflejar el problema de manera más cercana. Por lo cual, en el modelo computacional, se puede introducir una nueva población que represente la intervención de personal para que pueda frenar la propagación del contagio (Anderson, $1982 \mathrm{y}$ Wainer, 2018). Esto significa una nueva población en el sistema resultando en una nueva celda con reglas adicionales, para que se relacionen entre sí. Así mismo, sus consideraciones epidemiológicas y las reglas adicionales para el modelo computacional. La visión de esta introducción de la población de Soporte es prevenir la expansión de la enfermedad en la medida que se pueda. (Brauer y Castillo, 2001 y Wainer, 2002).

- La población de Soporte inmune a cualquier Enfermedad de Transmisión Sexual (ETS).

- La población de Soporte podría añadir Susceptibles a la población de Soporte mediante su presencia significativa y la influencia recibida de su vecindario (influencia positiva), y así poder conseguir la inmunidad. 
Por tanto, se definen nuevas reglas para el modelo computacional incluyendo la presencia de la población de Soporte . A esta población, se le asigna el número 4, y el color azul para la representación en la malla del autómata celular (Cell-DEVS). La introducción de esta nueva población intenta beneficiar a la reducción del contagio de la población Susceptible y no lleguen a pasar a la población de los Infectados (Pino et al., 2018 y Wainer, 2018).

La población de soporte sirve de apoyo a los susceptibles para que no contraigan la enfermedad (regla adicional), pero también a los infectados con ETS curable para que puedan regresar a la población susceptible (modificación de una regla) y se puedan mantener como una población inmune a la enfermedad según nuestras consideraciones porque se visualiza la erradicación del contagio irresponsable, y mantener en niveles bajos los infectados con ETS No curable y los que posee ambas y también que no haya brotes inesperados de la enfermedad (OMS, 2016). Un detalle importante es que en la realidad no existe una población de Soporte que brinde una inmunidad total, sino de prevención. Pero la perturbación nos permite visualizar la efectividad real que tiene tener una estrategia que pueda ser óptima para obtener una erradicación parcial de la epidemia. Sin embargo, la visualización que tiene una estrategia global permite tomar conciencia de lo esencial y primordial que es contar con una estrategia de prevención. (NIH, 2019 y Pino, 2017).

Tabla 5

Reglas adicionales de transición del modelo computacional

\begin{tabular}{ccc}
\hline $\begin{array}{c}\text { Estado inicial de la } \\
\text { célula }\end{array}$ & Regla para que se realice el cambio de estado & $\begin{array}{c}\text { Estado final de la } \\
\text { célula }\end{array}$ \\
0 & $\#(4) \geq 3 \wedge P b(\phi)$ & 4 \\
1 & $\#(2) \geq 1 \wedge \#(2)>\#(1) \wedge P b\left(\beta_{2}\right)$ & 0 \\
4 & {$[\#(2)+\#(3)=0 \vee \#(4) \geq 2] \wedge P b\left(\omega_{1}\right)$} & 4 \\
1 & Inmunidad & 3 \\
\hline
\end{tabular}

Hay que notar que la introducción de esta nueva celda muestra una actividad de prevención ante el contagio de la E.T.S. La presencia de esta nueva celda es ayudar de manera positiva, debido que en la expresión se cuantifica la influencia que recibe la celda central de su vecindario que puede ser positiva o negativa (Pino et al., 2018). Este detalle es importante debido que permite considerar una influencia positiva de un infectado o una influencia negativa de un susceptible y viceversa; esta consideración la modelamos con la función signo donde esta función tiene la propiedad de brindar bien un valor positivo o un valor negativo de forma aleatoria.
Se considera el mismo tamańo para ser introducida en la nuevacelda. La población total es de 2500 personas en la malla cuadrangular (), donde se distribuyen las poblaciones epidemiológicas. Las poblaciones de infectados permanecen con la misma cantidad inicial, y. La población de Susceptibles descenderá a 2175 personas, y la población de Soporte será de 75 personas para poder realizar la simulación computacional y poder analizar su evolución en el tiempo y espacio. 


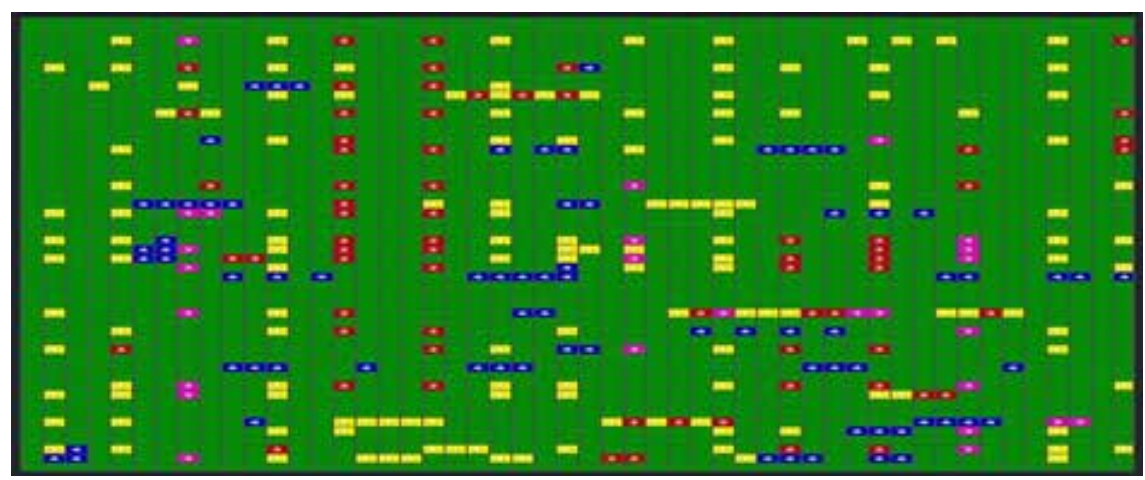

Figura 5. Estado inicial del Modelo Computacional

De forma similar al primer modelo, soporte para evaluar su impacto en las se propaga la enfermedad en el tiempo y espacio, a partir del estado inicial definido. Se ha introducido la nueva población de otras poblaciones epidemiológicas dentro de la malla de simulación. (Brauer y Castillo, 2001 y Pino et al., 2018).

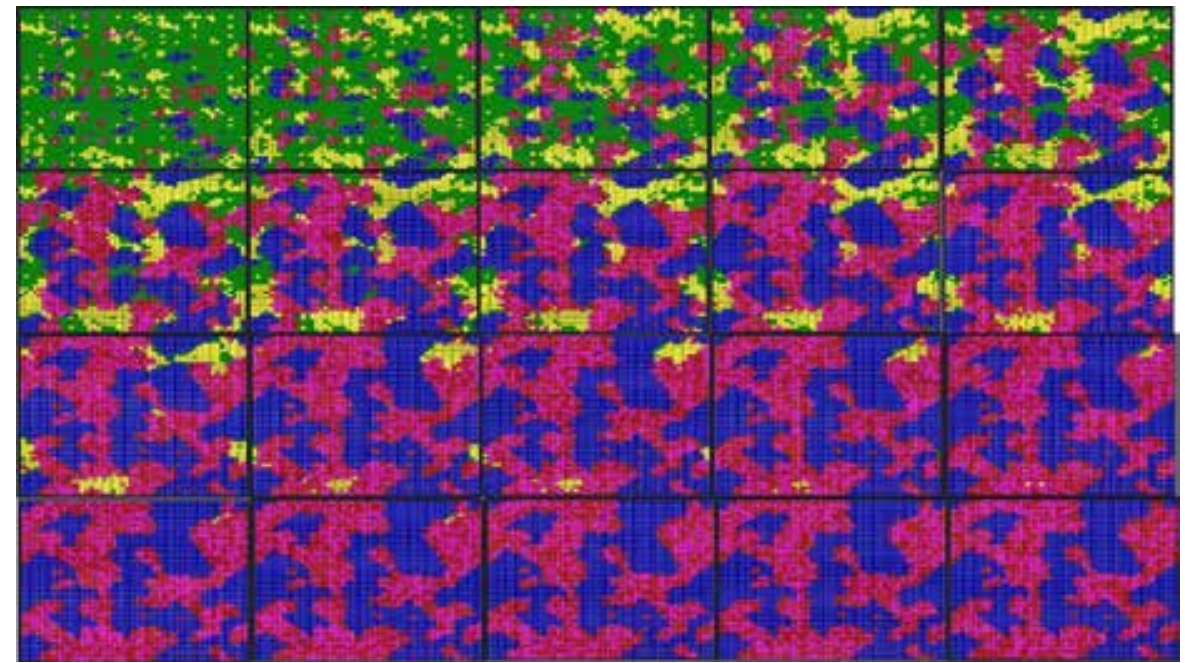

Figura 6. Evolución del Modelo Computacional en tiempo y espacio

De forma similar, se realiza una la evolución de las poblaciones en el gráfica (ploteo) considerando únicamente tiempo para analizarla.

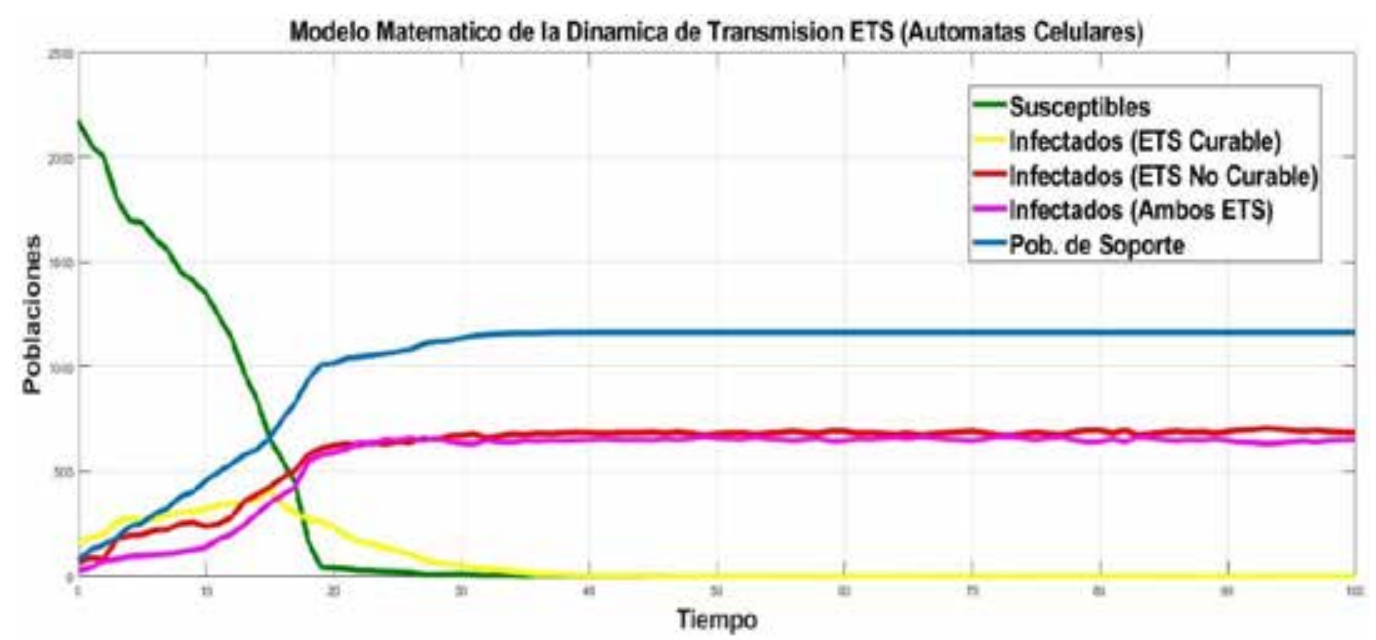

Figura 7. Evolución del Modelo Computacional en el tiempo 
Sin lugar a duda, la introducción de la población de Soporte ha suscitado un impacto positivo a las demás poblaciones. La población de Susceptibles ha descendido en ambas simulaciones, pero en la presente simulación su decrecimiento fue más rápido a causa de la población de Soporte (INS, 2015 y Ministerio de Salud, 2016) que lo ha ido incluyendo como una población inmune a la enfermedad (realización de métodos efectivos de protección) como también a las poblaciones de infectados. Finalmente, en ambas simulaciones computacionales, las poblaciones de los infectados (y mantienen una convivencia en el tiempo, pero en menor cantidad en la segunda simulación. (DGE, 2020). Por último, la población de infectados con ETS curable tuvo un crecimiento, pero luego comenzó a decrecer por la interacción con las demás poblaciones, de manera directa con las otras poblaciones de infectados. (NIH, 2019 y DGE, 2020).

\section{Conclusiones}

Los modelos que se han presentado aquí han permitido describir cómo se propagan las Enfermedades de Transmisión Sexual que son un problema de salud pública que posee el Ministerio de Salud donde tiene como prioridad disminuir el índice de contagio y su primer objetivo es la calidad de vida de cada ciudadano. En primer lugar, el modelamiento matemático aplicado a la epidemiología, en nuestro caso a las enfermedades de transmisión sexual, nos permite formular las consideraciones más importantes para la construcción del modelo. Los métodos numéricos nos permiten visualizar las soluciones aproximadas del sistema que se complementan con su análisis cualitativo (estabilidad de las soluciones). El margen de error reportado es significativo de y un tiempo de compilación de para el control de operaciones internas. (Bonaventura et al., 2013 y Wainer y Mosterman, 2016).

En segundo lugar y parte central de este trabajo, el modelamiento computacional por Autómatas celulares (Cell-DEVS) que mediante reglas sencillas se puede formular la evolución de la propagación de la enfermedad infecciosa (ETS). En la simulación computacional, se debe considerar el tiempo y el espacio a realizar debido que el costo operacional de los computadores es limitado. El tiempo de compilación es mucho más prolongado que en los métodos numéricos debido a la interacción de la celda central con su vecindario para cada celda de la malla de simulación durante el tiempo de simulación definido. (Pino et al., 2018 y Wainer, 2018). Sin lugar a duda, se tiene un trabajo de investigación que es aplicado a la epidemiología donde el modelamiento matemático permite generar proyecciones adecuadas en corto y mediano plazo para gestionar mejor los recursos que se tiene.

Además, la propuesta del modelo computacional por autómatas celulares necesita una construcción adecuada de las hipótesis y una formalización en los recursos informáticos que se requiere para que sean confiables los resultados. Por otro lado, la recolección de los datos siempre la realiza el Ministerio de Salud para estudiarla con las técnicas matemáticas y computacionales de tal manera que se puedan generar proyecciones que ayuden al sistema sanitario que se encarga de asistir a los pacientes con ETS. (OMS, 2015 y MINSA, 2019). Por lo cual, se 
puede concluir lo siguiente: (i) El modelo computacional mediante Autómatas celulares y realizado con el formalismo DEVS permite una mejor visualización de la manera cómo evoluciona la propagación de la enfermedad de transmisión sexual en tiempo y espacio.

La posición inicial de las personas infectadas nos permite analizar en qué ubicaciones se puede generar un brote más rápido y realizar intervenciones más oportunas dentro de la Salud Pública. Esto permite generar una mejor intervención epidemiológica como marca uno de los objetivos del plan estratégico del Ministerio de Salud. (ii) Las curvas poblaciones evidencian un escenario que se contrasta con los gráficos presentados por el Ministerio de Salud en cuanto a los años 2015, 2017 y 2019 donde se puede analizar un descenso considerable y a la vez un mejor posicionamiento de los centros de salud. Esto ayuda a seguir optimizando los recursos destinados. (iii) El modelamiento computacional por autómatas celulares permite generar escenarios aleatorios de un mismo estado inicial con lo cual se puede evaluar situaciones críticas de tal forma que ayude a focalizar la intervención y generar estrategias de prevención y control de las ETS.

Por otro lado, el modelamiento
matemático nos comportamiento temporal de las poblaciones consideradas de forma que generemos una serie de tiempo adecuada para evaluar cuantitativamente los esfuerzos realizados de año en año. (iv) El formalismo DEVS permite definir reglas sencillas de un sistema complejo (dinámica de la propagación de la ETS) con expresiones lógicas y realizar sus simulaciones computacionales en $\mathrm{CD}++$. Esto posibilita tener una aplicación accesible al personal médico que pueda visualizar y modificar el algoritmo de acuerdo a la situación que se presente para obtener curvas de proyección en tiempo real con la aplicación de reglas lógicas que conoce el personal de salud. (v) Existe un complemento entre la simulación realizada por los Métodos Numéricos, que describe la evolución de la enfermedad en el tiempo (EDO), y por los Autómatas Celulares (CellDEVS) que permiten un análisis ante la propagación de la enfermedad (tiempo y espacio); además que se obtiene una gráfica (ploteo) de las poblaciones en el tiempo para contrastar con la simulación de los métodos numéricos, y realizar mejores observaciones epidemiológicas e intervenciones en la salud pública.

Las simulaciones en el tiempo de los dos modelos permiten obtener conclusiones diferentes pero similares. La simulación que proporciona la interacción entre las poblaciones, donde la persona interactúa con todas las personas de cada población de acuerdo su tasa de contagio (métodos numéricos); y la que proporciona la interacción entre personas de acuerdo a su entorno determinado (autómatas celulares). Por ende, las aplicaciones epidemiológicas que permite son de una mayor apertura a tener recursos informáticos para evaluar casos y escenarios de forma de optimizar el análisis de intervención y control. 


\section{Referencias}

Anderson, R. (1982). Population Dynamics of Infectious Diseases: Theory and Application (1 ed.). Springer. DOI: https://doi.org/10.1007/978-14899-2901-3

Bonaventura, M., Wainer, G., \& Castro, R. (2013). Graphical modeling and simulation of discrete-event systems with $\mathrm{CD}_{++}$Builder. Simulation, 89(1), 4-27. DOI: https://doi. org/10.1177/0037549711436267

Brauer, F., \& Castillo-Chavez, C. (2001). Mathematical Models in Population Biology and Epidemiology. New York: Springer. DOI: https:// doi. org/10.1007/978-1-4614-1686-9

Centro Nacional de Epidemiología, P. y. (18 de marzo de 2020). Sala situacional para el Análisis de Situación de Salud 2020. (Ministerio de Salud, Productor) Obtenido de https:// www.dge.gob.pe/portal/index. php?option=com_content $\&$ view=category\&id $=2 \&$ Itemid $=204$

Centro Nacional de Epidemiología, P. y. (18 de marzo de 2020). Vigilancia Epidemiológica del VIH/SIDA. (Ministerio de Salud, Productor) Obtenido de https://www.dge.gob. pe/portal/index.php?option $=\mathrm{com}_{-}$ content $\&$ view $=$ article $\& i d=418 \& I-$ temid $=104$

Cueto, M. (2001). Culpa y Coraje: Historia de las Politicas sobre el VIH/ SIDA en el Perú. Lima, Perú: Consorcio de Investigación Económica y Social. Obtenido de https://www. cies.org.pe/es/publicaciones/diag- nostico-y-propuesta/culpa-y-coraje-historias-sobre-las-politicas-sobre-el-vihsida

Dirección General de Salud de las Personas. (2006). Guia Nacional de Consejería en ITS/VIH y el SIDA. Ministerio de Salud, Estrategia sanitaria Nacional Prevención y Control de Infecciones de Transmisión Sexual y VIH-SIDA, Lima. Obtenido de ftp://ftp2.minsa.gob.pe/ descargas/03esn/03itssida/Guias/ GuiaNacionalConsejeriaITS_VIH. pdf

Elkadry, A. (2013). Transmission Rate in Partial Differential Equation in Epidemic Models. Marshall University, Huntington. Obtenido de https://mds.marshall.edu/cgi/viewcontent.cgi? referer=https://www. google. $\mathrm{com} /$ \&httpsredir $=1$ \&article $=1543 \&$ context $=$ etd

Instituto Nacional de Estadística e Informática. (2009). Perú: Estimaciones y Proyecciones de Población por sexo, según departamento, provincia $y$ distrito, 2000-2015. Lima. Obtenido de https://proyectos.inei.gob.pe/ web/biblioineipub/bancopub/Est/ Lib0842/libro.pdf

Instituto Nacional de Salud. (2015). Anuario Estadístico 2015. Ministerio de Salud, Lima. Obtenido de http:// www.ins.gob.pe/insvirtual/images/ otrpubs/pdf/ANUARIO\%202015. pdf 
Kofman , E., \& Junco, S. (2001). Quantized-state systems: a DEVS Approach for continuous system simulation. Transactions of The Society for Modeling and Simulation International, 18(3), 123-132. DOI:https://dl.acm.org/ doi/10.5555/609891.609893

Ministerio de Salud. (2012). Boletín Estadistico sobre Infecciones sobre Infecciones de Transmisión Sexual Perú: 2002-2011. Oficina General de Estadística e Informática, Lima. Obtenido de http://bvs.minsa.gob. pe/local/MINSA/2728.pdf

Ministerio de Salud. (2015). Análisis de Situación de Salud de la Dirección de Salud IV Lima Este - 2015. Lima. Obtenido de http://v-beta.urp.edu. pe/pdf/id/21668/n/asis-2015-limaeste.pdf

Ministerio de Salud. (2017). Conocimiento de VIH e ITS. En Ministerio de Salud, Encuesta Demográfica y de Salud Familiar 2017 (pág. 24). Lima. Obtenido de https://www.inei.gob.pe/media/ MenuRecursivo/publicaciones_ digitales/Est/Lib1525/pdf/cap011. pdf

Ministerio de Salud. (2017). Situación de Salud de los Adolescentes y Jóvenes en el Perú. Lima. Obtenido de http://bvs.minsa.gob.pe/local/ MINSA/4143.pdf

Ministerio de Salud. (2019). Plan Multisectorial de Prevención y Control de las ITS/VIH y SIDA, 2015 - 2019. Ministerio de Salud,
Perú. Obtenido de ftp://ftp2. minsa.gob.pe/normaslegales/2015/ ANEXO_DEL_DECRETO_ SUPREMO_035-2015-SA.pdf

National Institutes of Health. (18 de marzo de 2020). Sexually Transmitted Diseases (STDs). (US Department of Health and Human Services, Productor) Obtenido de https://www.nichd.nih.gov/health/ topics/stds

Organización Mundial de la Salud. (2015). Estrategia Mundial del Sector de la Salud contra las Infecciones de Transmisión Sexual 2016-2021: hacia el Fin de las ITS. Obtenido de https://www.who.int/ reproductivehealth/STI_strategy_ abridged_es.pdf

Pino Romero, N. (2017). Análisis y simulación numérica de un modelo matemático SI con retardo discreto para las enfermedades de transmisión sexual. Universidad Nacional Mayor de San Marcos, Lima. Obtenido de http://cybertesis.unmsm.edu.pe/ handle/cybertesis/5735

Pino Romero, N., López Cruz, R., \& Wainer, G. (2018). Modelamiento computacional de la dinámica de transmisión sexual del VIH/SIDA mediante autómatas celulares (CellDEVS). Selecciones Matemáticas, 5(1), 39-47. DOI:http://dx.doi. org/10.17268/sel.mat.2018.01.06

Reynaga,C. (2015).Evaluación delimpacto de una campaña de prevención del VIH e ITS en adolescentes del Perú. Universitat Autónoma de Barcelona, Barcelona. Obtenido 
de https://www.researchgate. net/publication/284186129_ CARLOS_REYNAGA

Trottier, H., \& Philippe, P. (2000). Deterministic Modeling Of Infectious Diseases: Theory And Methods. The Internet Journal of Infectious Diseases, 1(2), 1-6. Obtenido de https://print.ispub. com/api/0/ispub-article/5230

Wainer, G. (1998). Discrete-Event cellular models with explicit delays. Université d'Aix-Marseille III.

Wainer, G. (2002). CD++ : a toolkit to develop DEVS models. Software: Practice and Experience, 32(13), 1261-1306. DOI:https://doi. org/10.1002/spe.482

Wainer, G. (2018). Advanced Cell-DEVS modeling applications: a legacy of Norbert Giambiasi. Simulation, $O(0), \quad$ 1-27. DOI:https://doi. org/10.1177/0037549718761596

Wainer, G., \& Castro, R. (2010). A Survey on the Application of the Cell-DEVS Formalism. Journal Cellular Automata, 5(6), 509-524.
Obtenido de https://pdfs.semanticscholar.org/dca6/cf39db32a3f9b3192c75e8ef00367f15df89. pdf

Wainer, G., \& Giambiasi, N. (2001). Application of the Cell-DEVS Paradigm for Cell Spaces Modelling and Simulation. Simulation, 76(1), 22-39. DOI:https:// doi.org/10.1177/003754970107 600102

Wainer, G., \& Mosterman, P. (2010). Discrete-Event Modeling and Simulation: Theory and Applications (1 ed.). CRC Press. Obtenido de https://www. crcpress.com/Discrete-EventModeling-and-SimulationTheory-and-Applications/ Wainer-Mosterman/p/ book/9781420072334

Wilchez Velásquez, C. (2014). Modelo de propagación de la malaria usando autómatas celulares con indicadores de probabilidad. Universidad de los Andres, Bogotá. Obtenido de https://repositorio.uniandes.edu. co/handle/1992/16431 